\title{
Perspective \\ Non-Covalent Interactions in Organic, Organometallic, and Inorganic Supramolecular Systems Relevant for Medicine, Materials Science, and Catalysis
}

\author{
Alexander S. Novikov
}

check for

updates

Citation: Novikov, A.S.

Non-Covalent Interactions in Organic, Organometallic, and Inorganic Supramolecular Systems Relevant for Medicine, Materials Science, and Catalysis. Crystals 2022, 12, 246. https://doi.org/10.3390/ cryst12020246

Academic Editor:

Jesús Sanmartín-Matalobos

Received: 27 January 2022

Accepted: 10 February 2022

Published: 11 February 2022

Publisher's Note: MDPI stays neutral with regard to jurisdictional claims in published maps and institutional affiliations.

Copyright: (c) 2022 by the author. Licensee MDPI, Basel, Switzerland. This article is an open access article distributed under the terms and conditions of the Creative Commons Attribution (CC BY) license (https:// creativecommons.org/licenses/by/ $4.0 /$ )
Institute of Chemistry, Saint Petersburg State University, Universitetskaya Nab., 7/9, 199034 Saint Petersburg, Russia; a.s.novikov@spbu.ru

\begin{abstract}
The structure, fundamental properties, and reactivity of chemical systems at various hierarchical levels of organization of matter is the paradigm of chemistry. A qualitative and quantitative description of various intermolecular and intramolecular non-covalent interactions in chemical systems is the main tool for supramolecular design and the driving force of smart prediction of kinetic and thermodynamic parameters of chemical reactions. This perspective is dedicated to highlighting the recent progress of our research group in the investigation of various non-covalent contacts in organic, organometallic, and inorganic chemical systems relevant for medicine, materials science, and catalysis. This research is interdisciplinary in nature and lies at the intersection of computer modeling with such natural science disciplines as chemistry, physics, crystallography, biology, and medicine, as well as directly related to materials science and nanotechnology.
\end{abstract}

Keywords: supramolecular systems; non-covalent contacts; weak interactions; crystal engineering; crystalline materials; crystal design and growth

The structure, fundamental properties, and reactivity of chemical systems at various hierarchical levels of organization of matter is the paradigm of chemistry. A qualitative and quantitative description of various intermolecular and intramolecular non-covalent interactions in chemical systems is the main tool for supramolecular design and the driving force of smart prediction of kinetic and thermodynamic parameters of chemical reactions. In the context of biochemistry, non-covalent interactions are necessary for the formation and folding of the three-dimensional structure of proteins and nucleic acids, as well as other macromolecules, ligand-protein binding. These inter- and intramolecular interactions have a significant effect on the properties of polymers, gels and membranes, on the dissolving abilities of liquids and their boiling points, and pharmaceutical and medicinal chemistry. The most common examples of such weak interactions are hydrogen, halogen, and chalcogen bonds and stacking interactions. Information about inter- and intramolecular interactions can be obtained experimentally, for example, based on X-ray diffraction analysis data, from phonon spectra and elastic characteristics of crystals, using thermogravimetric analysis and measurements of sublimation energies, and from spectral characteristics (for example, UV-Vis and IR spectroscopy, Raman spectroscopy, NMR spectroscopy). At the same time, these experimental methods provide only indirect information, largely mediated by experimental conditions, and often do not allow one to establish the nature (in particular, answer the question: are certain considered non-covalent contacts binding or repulsive?) and the energy of non-covalent interactions, which can be the goal and task for computer modeling and computational chemistry. Thus, the most adequate, comprehensive, and promising approach to the study of non-covalent interactions in chemical compounds and their supramolecular associates seems to be a combination of experimental observations and computer simulation results using the latest advances in quantum and computational chemistry. 
This perspective is dedicated to highlighting the recent progress of our research group in the combined experimental and theoretical investigation of various non-covalent contacts in organic, organometallic, and inorganic chemical systems relevant for medicine, materials science, and catalysis published in MDPI journals over the last 5 years. This research is interdisciplinary in nature and lies at the intersection of computer modeling with such natural science disciplines as chemistry, physics, crystallography, biology, and medicine, as well as directly related to materials science and nanotechnology.

In [1], the intra-/intermolecular bifurcated chalcogen bonds in thiazole/thiadiazole derived binuclear diaminocarbene complexes were discussed (Figure 1). The presence of such intermolecular chalcogen bonding leads to the fixation of an acetone molecule in the hollow between two diaminocarbene complexes in the solid state, the formation of dimeric associates or one-dimensional polymeric chains. These results are useful for understanding the relationship between the molecular structure and the crystal packing of systems with chalcogen bond donors and offer new opportunities in the crystal design of functional materials.

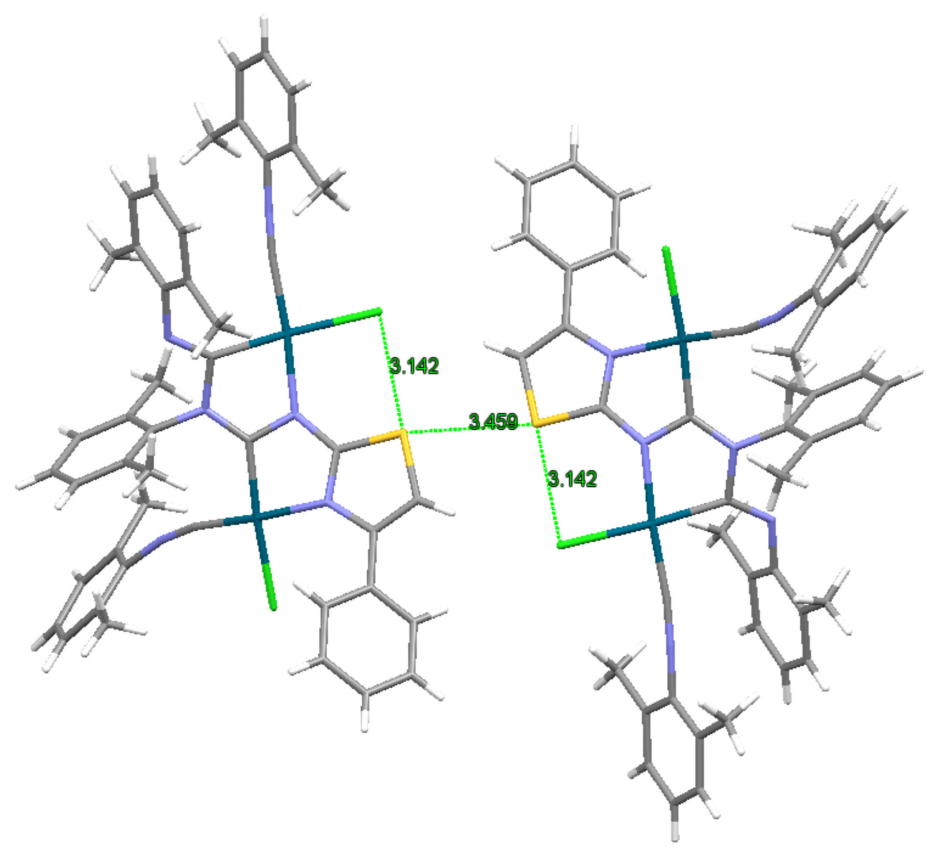

Figure 1. Intra-/intermolecular bifurcated chalcogen bonds in thiazole derived binuclear diaminocarbene complex.

In [2], we succeeded in co-crystallization of nevirapine (NVP, antiviral drug) with strong halogen bond donors, viz., 1,2,4,5-tetrafluoro-3,6-diiodobenzene (1,4-FIB) and 1,3diiodobenzene (1,3-DIB) (Figure 2). In the X-ray structures of NVP·1,4-FIB and NVP·1,3DIB co-crystals, various supramolecular contacts (viz., hydrogen and halogen bonds) were detected and further investigated via quantum chemical modeling. Moreover, in [3], we identified an opportunity for co-crystallization of anastrozole (ASZ) with 1,4-FIB. In the X-ray structure of ASZ-1.5 (1,4-FIB) co-crystal, different supramolecular interactions (viz., hydrogen and halogen bonding) were found and studied via DFT calculations. These findings continue to provide proof of principle for the productive employment of noncovalent interactions in the discovery of stable crystalline forms of drug substances for the medical industry. 


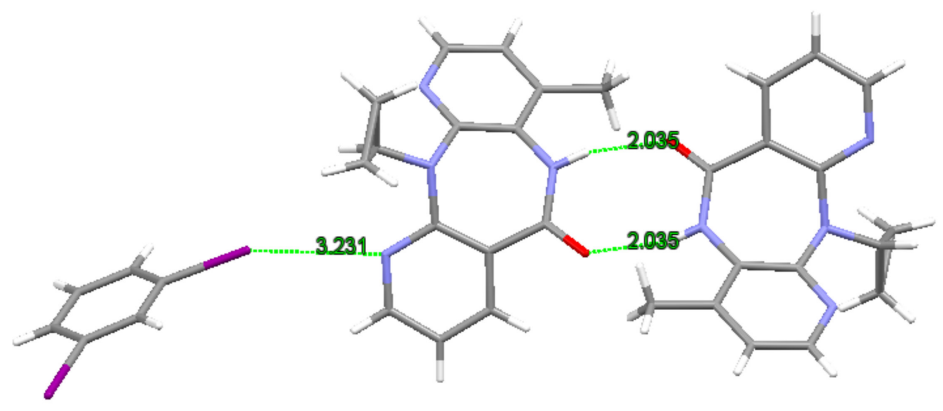

Figure 2. Supramolecular contacts (viz., hydrogen and halogen bonds) in NVP.1,3-DIB co-crystal.

In [4], a series of halogen-bonded complexes formed by $\mathrm{O}=\mathrm{PMe}_{3}$ and various halogencontaining molecules were studied by quantum chemical calculations. The results reveal correlations between changes of spectroscopic parameters of phosphine oxide upon complexation, such as ${ }^{31} \mathrm{P}$ NMR chemical shift, $\mathrm{P}=\mathrm{O}$ stretching frequency, quantum theory of atoms in molecules (QTAIM) parameters at halogen bond critical point, halogen's sigma-hole size, $\mathrm{X} \cdots \mathrm{O}$ distance, and $\mathrm{R}-\mathrm{X} \cdots \mathrm{O}=\mathrm{PMe}_{3}$ halogen bond energy. The proposed correlations could be used to estimate the halogen bonding properties in glasses and solutions from the corresponding IR and NMR spectra.

In [5], the 16-membered cyclic heterooctamer formed via halogen bonds between the $\mathrm{SCN}^{-}$and iodonium cation in the crystal of 4-methoxyphenyl(phenyl)iodonium thiocyanate, $\left[\mathrm{PhI}\left(4-\mathrm{C}_{6} \mathrm{H}_{4} \mathrm{OMe}\right)\right](\mathrm{SCN})$, was reported and studied theoretically by quantum chemical calculations (Figure 3). Our search in the Cambridge Structural Database results in the identification of only three examples of similar heterooctamer clusters containing $\mathrm{SCN}^{-}$or iodonium cations. Thus, our work shows that such heterooctamer clusters containing $\mathrm{SCN}^{-}$or iodonium cations could be potentially interesting supramolecular motifs for crystal engineering.

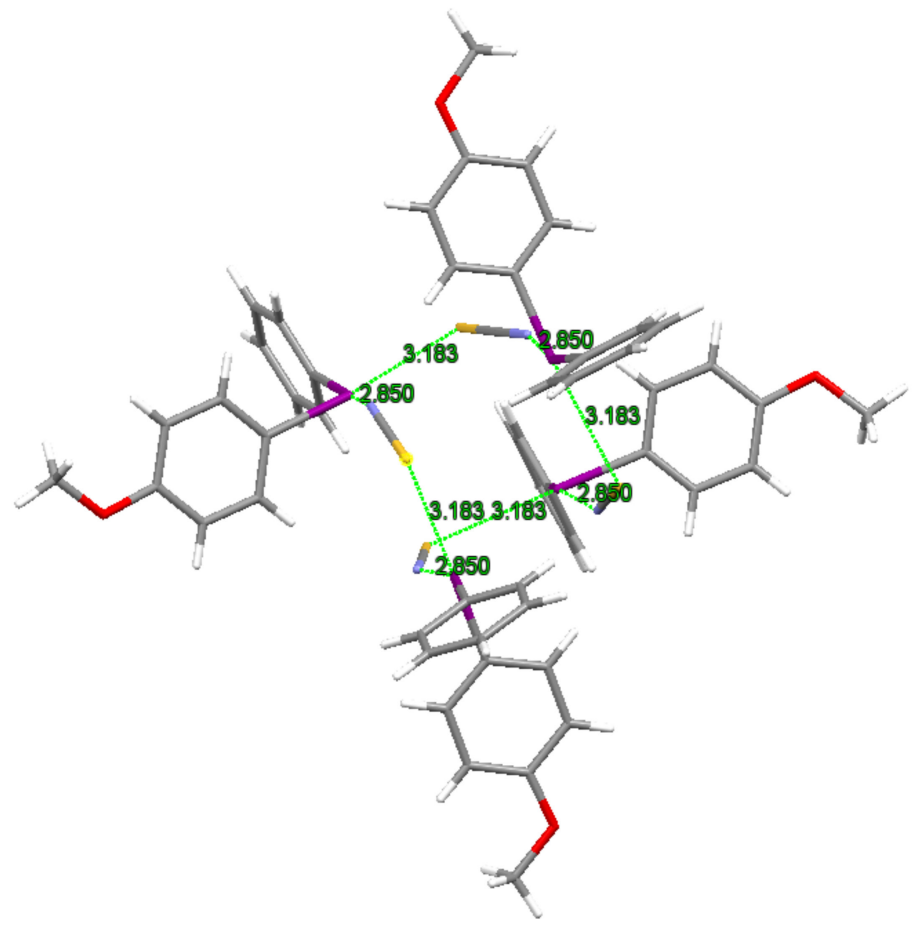

Figure 3. 16-membered cyclic heterooctamer formed via halogen bonds between the $\mathrm{SCN}^{-}$ and iodonium cation in the crystal of 4-methoxyphenyl(phenyl)iodonium thiocyanate, [PhI(4$\left.\left.\mathrm{C}_{6} \mathrm{H}_{4} \mathrm{OMe}\right)\right](\mathrm{SCN})$. 
In [6], [Co(2-XPy $\left.)_{2} \mathrm{Cl}_{2}\right](\mathrm{X}=\mathrm{Cl}, \mathrm{Br}$, and I) isostructural complexes (Figure 4) were synthesized and characterized. The strength of halogen bonding in a solid state was studied using quantum chemical calculations, revealing the halogen bond's energy growth from $\mathrm{Cl}$ to $\mathrm{I}$. In our opinion, such $\mathrm{N}$-donor halogen-substituted ligands are promising potential building blocks for crystal engineering and material science.

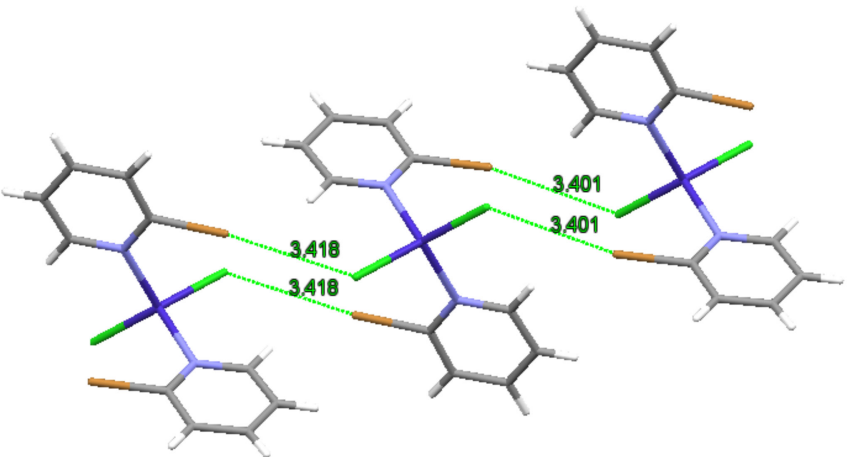

Figure 4. Halogen bonding in crystal structure of $\left[\mathrm{Co}\left(2-\mathrm{BrPy}_{2} \mathrm{Cl}_{2}\right]\right.$ complexes.

In [7], we showed that the cyclopentanone reacts with 4-bromobenzamidrazone giving 3-(4-bromophenyl)-5-(4-peroxobutyl)-1,2,4-triazole, which future formed pale-yellow crystals featuring attractive intermolecular non-covalent interactions $\mathrm{Br} \cdots \mathrm{Br}$ (type I halogen $\cdots$ halogen contacts). These weak contacts stabilize this peroxo compound in the solid state and are suggested to be the driving forces for crystallization (Figure 5). Thus, such halogen bonds could be used as "supramolecular staples" to stabilize unstable compounds.

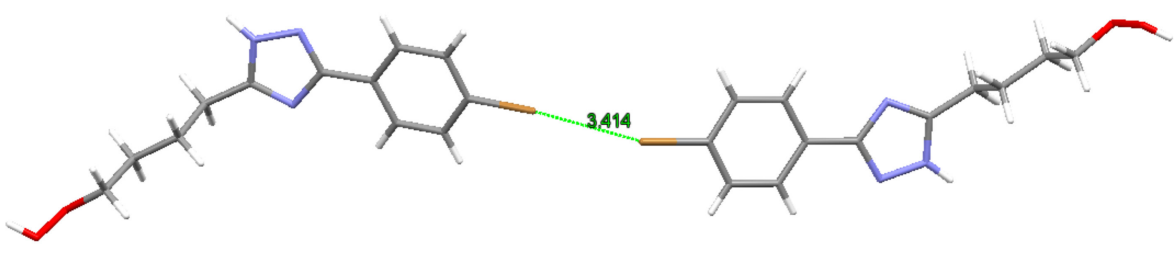

Figure 5. Intermolecular non-covalent interactions $\mathrm{Br} \cdots \mathrm{Br}$ (type I halogen $\cdots$ halogen contacts) in crystal of 3-(4-bromophenyl)-5-(4-peroxobutyl)-1,2,4-triazole.

In [8], a series of halogenated aromatic dichlorodiazadienes were synthesized. Multiple weak halogen $\cdots$ halogen contacts were fixed in the solid state (Figure 6) and studied by quantum chemical calculations. Theoretical studies demonstrated that non-covalent halogen $\cdots$ halogen interactions play a crucial role in the self-assembly of highly polarizable dichlorodiazadienes-a novel class of azo-dyes.

In [9], novel members of the 1,2-diboraoxazoles family were obtained in mild conditions and with good yields via an intramolecular ring-closure reaction of borylated iminols $\left[\mathrm{B}_{10} \mathrm{H}_{9} \mathrm{~N}=\mathrm{C}(\mathrm{OH}) \mathrm{R}\right]^{-}$. The bonding interactions in the 1,2-diboraoxazole cycles were theoretically studied by quantum chemical calculations and QTAIM analysis. Such boron clusters are of potential interest in boron neutron-capture cancer therapy.

In [10], we showed that the interaction between 2-halogen-substituted pyridines (2-XPy) and $\mathrm{Zn}(\mathrm{II})$ dihalides leads to the formation of a series of heteroleptic molecular complexes [(2-Xpy $\left.)_{2} \mathrm{ZnY}_{2}\right](\mathrm{X}, \mathrm{Y}=\mathrm{Cl}, \mathrm{Br}$, and I). Most of these heteroleptic molecular complexes [(2-Xpy $\left.)_{2} \mathrm{ZnY}_{2}\right]$ are isostructural (triclinic), while two of them are monoclinic. In all cases, halogen bonding plays a dramatic role in crystal packing (Figure 7). Moreover, many of these heteroleptic molecular complexes [(2-Xpy $\left.{ }_{2} \mathrm{ZnY}_{2}\right]$ feature luminescence in a solid state (quantum yields exceed $21 \%$ ), which could be interesting for materials science. 


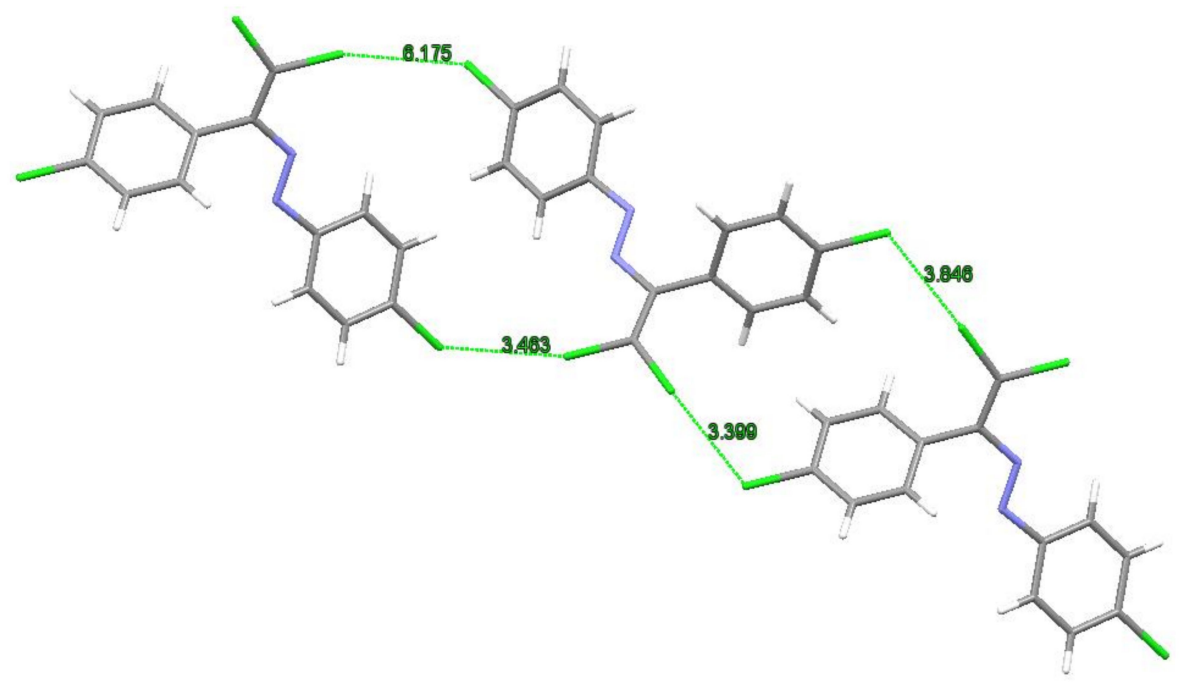

Figure 6. Multiple $\mathrm{Cl} \cdots \mathrm{Cl}$ contacts in halogenated aromatic dichlorodiazadiene.

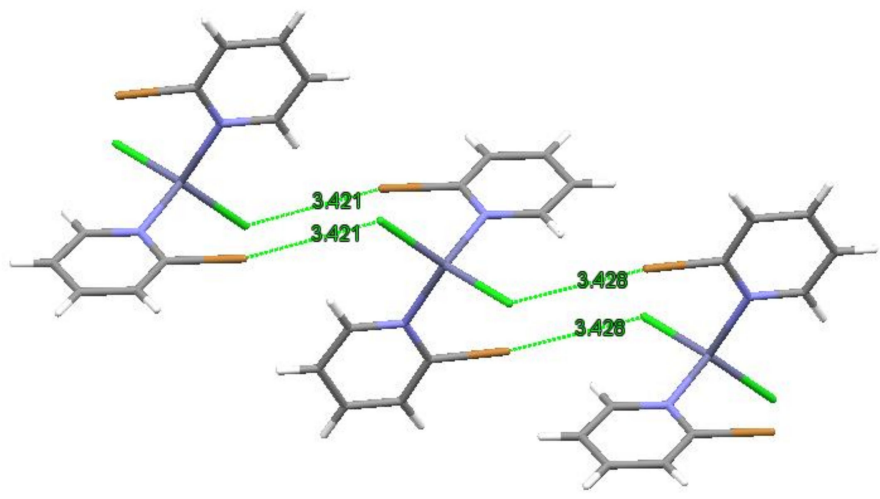

Figure 7. Halogen bonding $\mathrm{Br} \cdots \mathrm{Cl}$ in heteroleptic molecular complex $\left[\left(2-\mathrm{BrPy}_{2} \mathrm{ZnCl}_{2}\right]\right.$.

In [11], we have studied the encapsulation of Rhodamine 6G dye molecules in a melamine barbiturate robust host system via supramolecular assembly. The encapsulation of the dye was investigated by scanning electron and optical fluorescent microscopies, UV-Vis spectroscopy, single crystal, and powder X-ray diffraction. Additionally, molecular dynamics and DFT calculations were utilized for modeling self-assembly processes in the studied system. We found that the encapsulation of Rhodamine 6G molecules inside the melamine barbiturate robust host system leads to the dramatic enhancement in the organic crystal's shape, lesser degree of twinning, and higher symmetry.

In [12], N-pyridyl ureas with 1,2,4- and 1,3,4-oxadiazole moieties featuring the (oxadiazole) ...(pyridine) and (oxadiazole) ...(oxadiazole) non-covalent interactions (Figure 8) were synthesized. The presence of these interactions was confirmed theoretically by quantum chemical calculations, including non-covalent interactions (NCI) and QTAIM analysis. The database survey allowed the verification of additional examples of relevant (oxadiazole) $\cdots \pi$ interactions both in the Protein Data Bank and Cambridge Structural Database, including the co-crystal of commercial anti-HIV drug Raltegravir. Thus, such non-covalent interactions (oxadiazole) $\cdots$ (pyridine) and (oxadiazole) $\cdots$ (oxadiazole) could be essential in solid form of active pharmaceutical ingredients.

In [13], the $\mathrm{Br}_{2}$ oxidative cleavage of the $\mathrm{Te}-\mathrm{Te}$ bond of dipyridylditelluride leading to the formation of 2-pyridyltellurenyl bromide is reported. The Te atom of 2-pyridyltellurenyl bromide is involved in four different non-covalent contacts forming a 3D supramolecular symmetrical framework: one $\mathrm{Te} \cdots \mathrm{N}$ chalcogen bond, two $\mathrm{Te} \cdots \mathrm{Br}$ chalcogen bonds, and $\mathrm{Te} \cdots \mathrm{Te}$ interactions. Such novel building blocks could be interesting and promising for crystal engineering and design. 


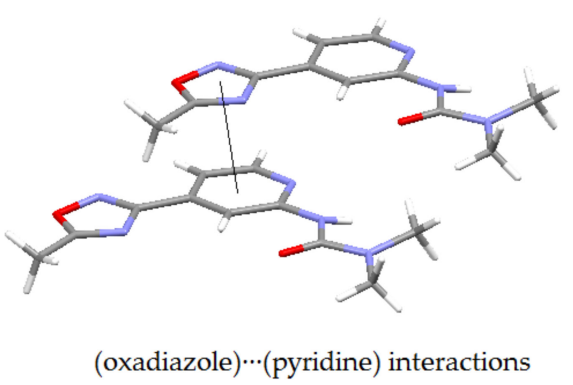

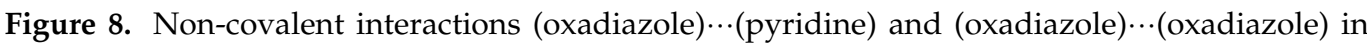
$\mathrm{N}$-pyridyl ureas with 1,2,4- and 1,3,4-oxadiazole moieties.

Finally, in [14], the synthesis of borylated amidines based on the closo-dodecaborate anion $\left[\mathrm{B}_{12} \mathrm{H}_{11} \mathrm{NCCH}_{3} \mathrm{NHR}\right]^{-}(\mathrm{R}=\mathrm{H}$, Alk, and Ar) was developed. The mechanism of the addition of amine to the nitrile derivative of the closo-dodecaborate anion was studied using DFT calculations. The structure of the transition state and appropriate non-covalent interactions determine the stereoselectivity of the reaction. The screening of the biological properties of borylated amidine sodium salts indicated that the substances could accumulate in cancer cells in significant amounts and had a low toxicity.

Thus, non-covalent interactions are one of the hottest research areas in modern chemistry and a number of relatively recent thematic issues of the most authoritative review chemical journal Chem. Rev. [15-18] were completely devoted to the phenomenon of noncovalent interactions. Additionally, there are several excellent review articles focused on covalent organic frameworks (highly porous and crystalline polymeric materials, constructed by covalent bonds and extending in two or three dimensions), in particular, triazine derivatives [19], which could be new and promising materials platforms for photocatalytic $\mathrm{CO}_{2}$ reduction [20] and the degradation of aqueous pollutants [21]; various non-covalent interactions can play a very important role in such processes.

From my point of view, the following key challenging points and future development directions on the application of non-covalent interactions in single-crystal organic, organometallic, and inorganic compounds could be emphasized: creating building blocks capable of bonding with controllable and predictable strength and orientation; the creation of polyfunctional building blocks; the development of most cost-effective building blocks; the manufacturing of sustainable and environmentally friendly building blocks.

I believe that other authors and research groups will follow my initiative in publishing articles in Crystals regarding their most recent and important achievements in the fields of supramolecular chemistry and non-covalent interactions, and readers will have the opportunity to briefly receive the latest and most up-to-date information about this modern topic.

Funding: This perspective article was written without receiving any external funding.

Conflicts of Interest: The author declares no conflict of interest.

\section{References}

1. Mikherdov, A.S.; Novikov, A.S.; Kinzhalov, M.A.; Zolotarev, A.A.; Boyarskiy, V.P. Intra-/Intermolecular Bifurcated Chalcogen Bonding in Crystal Structure of Thiazole/Thiadiazole Derived Binuclear (Diaminocarbene)PdII Complexes. Crystals $2018,8,112$. [CrossRef]

2. Kryukova, M.A.; Sapegin, A.V.; Novikov, A.S.; Krasavin, M.; Ivanov, D.M. New Crystal Forms for Biologically Active Compounds. Part 1: Noncovalent Interactions in Adducts of Nevirapine with XB Donors. Crystals 2019, 9, 71. [CrossRef]

3. Kryukova, M.A.; Sapegin, A.V.; Novikov, A.S.; Krasavin, M.; Ivanov, D.M. New Crystal Forms for Biologically Active Compounds. Part 2: Anastrozole as N-Substituted 1,2,4-Triazole in Halogen Bonding and Lp- $\pi$ Interactions with 1,4-Diiodotetrafluorobenzene. Crystals 2020, 10, 371. [CrossRef] 
4. Ostras', A.S.; Ivanov, D.M.; Novikov, A.S.; Tolstoy, P.M. Phosphine Oxides as Spectroscopic Halogen Bond Descriptors: IR and NMR Correlations with Interatomic Distances and Complexation Energy. Molecules 2020, 25, 1406. [CrossRef]

5. Soldatova, N.S.; Suslonov, V.V.; Kissler, T.Y.; Ivanov, D.M.; Novikov, A.S.; Yusubov, M.S.; Postnikov, P.S.; Kukushkin, V.Y. Halogen Bonding Provides Heterooctameric Supramolecular Aggregation of Diaryliodonium Thiocyanate. Crystals 2020, 10, 230. [CrossRef]

6. Adonin, S.A.; Bondarenko, M.A.; Novikov, A.S.; Sokolov, M.N. Halogen Bonding in Isostructural Co(II) Complexes with 2-Halopyridines. Crystals 2020, 10, 289. [CrossRef]

7. Bolotin, D.S.; Il'in, M.V.; Suslonov, V.V.; Novikov, A.S. Symmetrical Noncovalent Interactions Br-Br Observed in Crystal Structure of Exotic Primary Peroxide. Symmetry 2020, 12, 637. [CrossRef]

8. Nenajdenko, V.G.; Shikhaliyev, N.G.; Maharramov, A.M.; Bagirova, K.N.; Suleymanova, G.T.; Novikov, A.S.; Khrustalev, V.N.; Tskhovrebov, A.G. Halogenated Diazabutadiene Dyes: Synthesis, Structures, Supramolecular Features, and Theoretical Studies. Molecules 2020, 25, 5013. [CrossRef]

9. Voinova, V.V.; Selivanov, N.A.; Plyushchenko, I.V.; Vokuev, M.F.; Bykov, A.Y.; Klyukin, I.N.; Novikov, A.S.; Zhdanov, A.P.; Grigoriev, M.S.; Rodin, I.A.; et al. Fused 1,2-Diboraoxazoles Based on closo-Decaborate Anion-Novel Members of Diboroheterocycle Class. Molecules 2021, 26, 248. [CrossRef]

10. Vershinin, M.; Rakhmanova, M.; Novikov, A.; Sokolov, M.; Adonin, S. Zn(II) Heteroleptic Halide Complexes with 2-Halopyridines: Features of Halogen Bonding in Solid State. Molecules 2021, 26, 3393. [CrossRef]

11. Nesterov, P.; Shilovskikh, V.; Sokolov, A.; Gurzhiy, V.; Novikov, A.; Timralieva, A.; Belogub, E.; Kondratyuk, N.; Orekhov, N.; Skorb, E. Encapsulation of Rhodamine 6G Dye Molecules for Affecting Symmetry of Supramolecular Crystals of Melamine-Barbiturate. Symmetry 2021, 13, 1119. [CrossRef]

12. Baykov, S.V.; Mikherdov, A.S.; Novikov, A.S.; Geyl, K.K.; Tarasenko, M.V.; Gureev, M.A.; Boyarskiy, V.P. $\pi-\pi$ Noncovalent Interaction Involving 1,2,4- and 1,3,4-Oxadiazole Systems: The Combined Experimental, Theoretical, and Database Study. Molecules 2021, 26, 5672. [CrossRef] [PubMed]

13. Buslov, I.V.; Novikov, A.S.; Khrustalev, V.N.; Grudova, M.V.; Kubasov, A.S.; Matsulevich, Z.V.; Borisov, A.V.; Lukiyanova, J.M.; Grishina, M.M.; Kirichuk, A.A.; et al. 2-Pyridylselenenyl versus 2-Pyridyltellurenyl Halides: Symmetrical Chalcogen Bonding in the Solid State and Reactivity towards Nitriles. Symmetry 2021, 13, 2350. [CrossRef]

14. Nelyubin, A.V.; Selivanov, N.A.; Bykov, A.Y.; Klyukin, I.N.; Novikov, A.S.; Zhdanov, A.P.; Karpechenko, N.Y.; Grigoriev, M.S.; Zhizhin, K.Y.; Kuznetsov, N.T. Primary Amine Nucleophilic Addition to Nitrilium Closo-Dodecaborate [B12H11NCCH3]-: A Simple and Effective Route to the New BNCT Drug Design. Int. J. Mol. Sci. 2021, 22, 13391. [CrossRef] [PubMed]

15. Huang, F.; Anslyn, E.V. Introduction: Supramolecular Chemistry. Chem. Rev. 2015, 115, 6999-7000. [CrossRef]

16. Percec, V. Introduction to Frontiers in Macromolecular and Supramolecular Science: Part 1. Chem. Rev. 2016, 116, 769-770. [CrossRef]

17. Percec, V. Introduction to Frontiers in Macromolecular and Supramolecular Science: Part 2. Chem. Rev. 2016, 116, 1671-1672. [CrossRef]

18. Hobza, P.; Řezáč, J. Introduction: Noncovalent Interactions. Chem. Rev. 2016, 116, 4911-4912. [CrossRef]

19. Zhang, Y.; Jin, S. Recent Advancements in the Synthesis of Covalent Triazine Frameworks for Energy and Environmental Applications. Polymers 2018, 11, 31. [CrossRef]

20. Li, J.; Zhao, D.; Liu, J.; Liu, A.; Ma, D. Covalent Organic Frameworks: A Promising Materials Platform for Photocatalytic CO 2 Reductions. Molecules 2020, 25, 2425. [CrossRef]

21. Qian, Y.; Ma, D. Covalent Organic Frameworks: New Materials Platform for Photocatalytic Degradation of Aqueous Pollutants. Materials 2021, 14, 5600. [CrossRef] [PubMed] 